\title{
Path Choosability of Planar Graphs
}

\author{
Glenn G. Chappell \\ Department of Computer Science \\ University of Alaska \\ Fairbanks, AK, U.S.A. \\ ggchappell@alaska.edu
}

\author{
Chris Hartman \\ Department of Computer Science \\ University of Alaska \\ Fairbanks, AK, U.S.A. \\ cmhartman@alaska.edu
}

Submitted: Jul 7, 2017; Accepted: Sep 24, 2018; Published: Nov 16, 2018

(c) The authors. Released under the CC BY license (International 4.0).

\begin{abstract}
A path coloring of a graph $G$ is a vertex coloring of $G$ such that each color class induces a disjoint union of paths. We consider a path-coloring version of list coloring for planar and outerplanar graphs. We show that if each vertex of a planar graph is assigned a list of 3 colors, then the graph admits a path coloring in which each vertex receives a color from its list. We prove a similar result for outerplanar graphs and lists of size 2 .

For outerplanar graphs we prove a multicoloring generalization. We assign each vertex of a graph a list of $q$ colors. We wish to color each vertex with $r$ colors from its list so that, for each color, the set of vertices receiving it induces a disjoint union of paths. We show that we can do this for all outerplanar graphs if and only if $q / r \geqslant 2$. For planar graphs we conjecture that a similar result holds with $q / r \geqslant 3$; we present partial results toward this conjecture.
\end{abstract}

Mathematics Subject Classifications: Primary 05C15; Secondary 05C10

\section{Introduction}

All graphs will be finite, simple, and undirected. See West [27] for graph theoretic terms.

We denote the vertex set of a graph $G$ by $V(G)$. A path coloring of $G$ is a coloring (not necessarily proper) of $V(G)$ such that each color class induces a linear forest, that is, a forest of maximum degree at most 2, or, equivalently, a disjoint union of paths. A graph $G$ is path $k$-colorable if $G$ admits a path coloring using $k$ colors.

Every outerplanar graph is path 2-colorable; this was proven independently by Broere \& Mynhardt [7, Thm. 3] and by Akiyama, Era, Gervacio, \& Watanabe [1, Thm. 2.3].

Theorem 1.1 (Broere \& Mynhardt 1985; Akiyama, Era, Gervacio, \& Watanabe 1989). If $G$ is an outerplanar graph, then $G$ is path 2-colorable. 
Chartrand \& Kronk [10, Section 3] gave an example of a planar graph whose vertex set cannot be partitioned into two subsets, each inducing a forest. It follows that not every planar graph is path 2-colorable. Broere \& Mynhardt conjectured [7, Conj. 16] that every planar graph is path 3-colorable. This was proven independently by Poh [22, Thm. 2] and by Goddard [14, Thm. 1].

Theorem 1.2 (Poh 1990, Goddard 1991). If $G$ is a planar graph, then $G$ is path 3colorable.

Path colorability has a natural list-coloring generalization. A graph $G$ is path $k$ choosable if, whenever each vertex of $G$ is assigned a list of $k$ colors, there exists a path coloring of $G$ in which each vertex receives a color from its list.

A related, slightly weaker notion that has led to a number of results is that of 2defective choosability. A graph $G$ is $t$-defective $k$-choosable if, whenever each vertex of $G$ is assigned a list of $k$ colors, there exists a vertex coloring of $G$ in which each vertex receives a color from its list, and each color class induces a subgraph of maximum degree at most $t$. When $t=2$, each color class is a disjoint union of paths and cycles.

Much of the work on these types of list coloring has looked at restricted classes of planar graphs. Of particular interest have been questions involving lists of size 2 .

One way to restrict the class of planar graphs is to place a lower bound on the girth. Škrekovski [24, Thm. 1.1] showed that each planar graph of girth at least 7 is 2-defective 2-choosable. Havet \& Sereni [18, Corollary 1] improved this by showing by showing that girth at least 6 suffices. Chappell, Gimbel, \& Hartman [9, Thm. 3.1] further improved this by showing that each planar graph of girth at least 6 is path 2-choosable. And Axenovich, Ueckerdt, \& Weiner [5, Thm. 1] improved the Chappell-Gimbel-Hartman result by showing the existence of a path coloring in which each monochromatic component is a path on at most 15 vertices.

In another improvement of the Škrekovski result, Borodin \& Ivanova [6, Thm. 1] showed that each planar graph of girth at least 7 is path 2-choosable such that, in the path coloring, each monochromatic component is a path on at most 3 vertices.

In the other direction, Škrekovski [23, Prop. 3.2] constructed a planar graph of girth 4 that is not 2-defective 2-choosable (also see Chappell, Gimbel, \& Hartman [9, Corollary 3.4]). So none of the above girth-related results can be extended to planar graphs of girth 4 . The question of whether every planar graph of girth 5 is path 2-choosable, or 2-defective 2-choosable, remains open.

We can also restrict planar graphs by placing bounds on the maximum degree. Chappell, Gimbel, \& Hartman [9, Corollary 2.5, Prop. 2.7] showed that every planar graph with maximum degree at most 4 is path 2-choosable, while there exists a planar graph with maximum degree 5 that has no path 2-coloring.

Concerning planar graphs in general, Škrekovski [23, Thm. 2.2b] and Eaton \& Hull [12, Thm. 1] independently showed that every planar graph is 2-defective 3-choosable.

Defective choosability has also been investigated for various other classes of graphs, including graphs on surfaces of higher genus (see Woodall [29]) and graphs with bounded maximum average degree (see Havet \& Sereni [18]). Recently, Ossona de Mendez, Oum, 
\& Wood [21] investigated defective choosability for classes of graphs with certain excluded subgraphs, leading to new results for a number of graph classes. See Wood [28] for a survey of defective coloring.

In this work, we investigate list-coloring generalizations of Theorems 1.1 and 1.2.

In Section 2, we show that "colorable" may be replaced by "choosable" in the statements of Theorems 1.1 and 1.2: every outerplanar graph is path 2-choosable, and every planar graph is path 3-choosable. Thus, we strengthen the results of Škrekovski [23, Thm. 2.2b] and Eaton \& Hull [12, Thm. 1].

Erdős, Rubin, \& Taylor [13] introduced a generalization of ordinary list coloring. A graph $G$ is $(q, r)$-choosable, for positive integers $q$ and $r$, if, whenever each vertex of $G$ is assigned a list of $q$ colors, we may color each vertex with a set of $r$ colors from its list so that adjacent vertices are colored with disjoint sets.

We define a path-coloring version of this generalized list coloring. In a path multicoloring of a graph $G$ each vertex of $G$ is colored with a set of colors such that, for each color, the collection of vertices whose set contains that color, induces a linear forest. Note that, if each vertex is colored with a set of size 1, then the result is an ordinary path coloring. A graph $G$ is path $(q, r)$-choosable if, whenever each vertex of $G$ is assigned a list of $q$ colors, there is a path multicoloring of $G$ in which each vertex receives a set of $r$ colors from its list. This generalizes our earlier definition of path choosability, as path $(k, 1)$-choosability is the same as path $k$-choosability.

In Section 3, we consider path $(q, r)$-choosability of outerplanar graphs. We show that every outerplanar graph is path $(q, r)$-choosable iff $q / r \geqslant 2$, for $q$ and $r$ positive integers.

In Section 4, we conjecture that a similar result holds for planar graphs with $q / r \geqslant$ 3 ; we present partial results toward this conjecture. In particular, we show that the implication holds in one direction: if every planar graph is path $(q, r)$-choosable, then $q / r \geqslant 3$. We also show that, for each planar graph $G$, there exist an infinite number of positive integers $m$ such that $G$ is path $(3 m, m)$-choosable.

\section{Path Choosability}

We can generalize Theorem 1.2 to path choosability.

Theorem 2.1. If $G$ is a planar graph, then $G$ is path 3-choosable.

Theorem 2.1 was proven by the second author in his Ph.D. thesis [17, Thm. 4.1]. Our proof is based on the proof found there, with some ideas taken from a proof by Škrekovski [23, pf. of Lemma 2.1] of a slightly different result, using essentially the same technique.

Theorem 2.1 follows from our next lemma. By a planar graph we mean a graph that can be drawn in the plane without crossing edges, while a plane graph is a planar graph along with an embedding in the plane. Given $x, y \in V(G)$, we denote by $S_{G}(x, y)$ the set containing $x, y$, and all cut vertices of $G$ that separate $x$ and $y$; in particular, $S_{G}(x, x)=\{x\}$. 


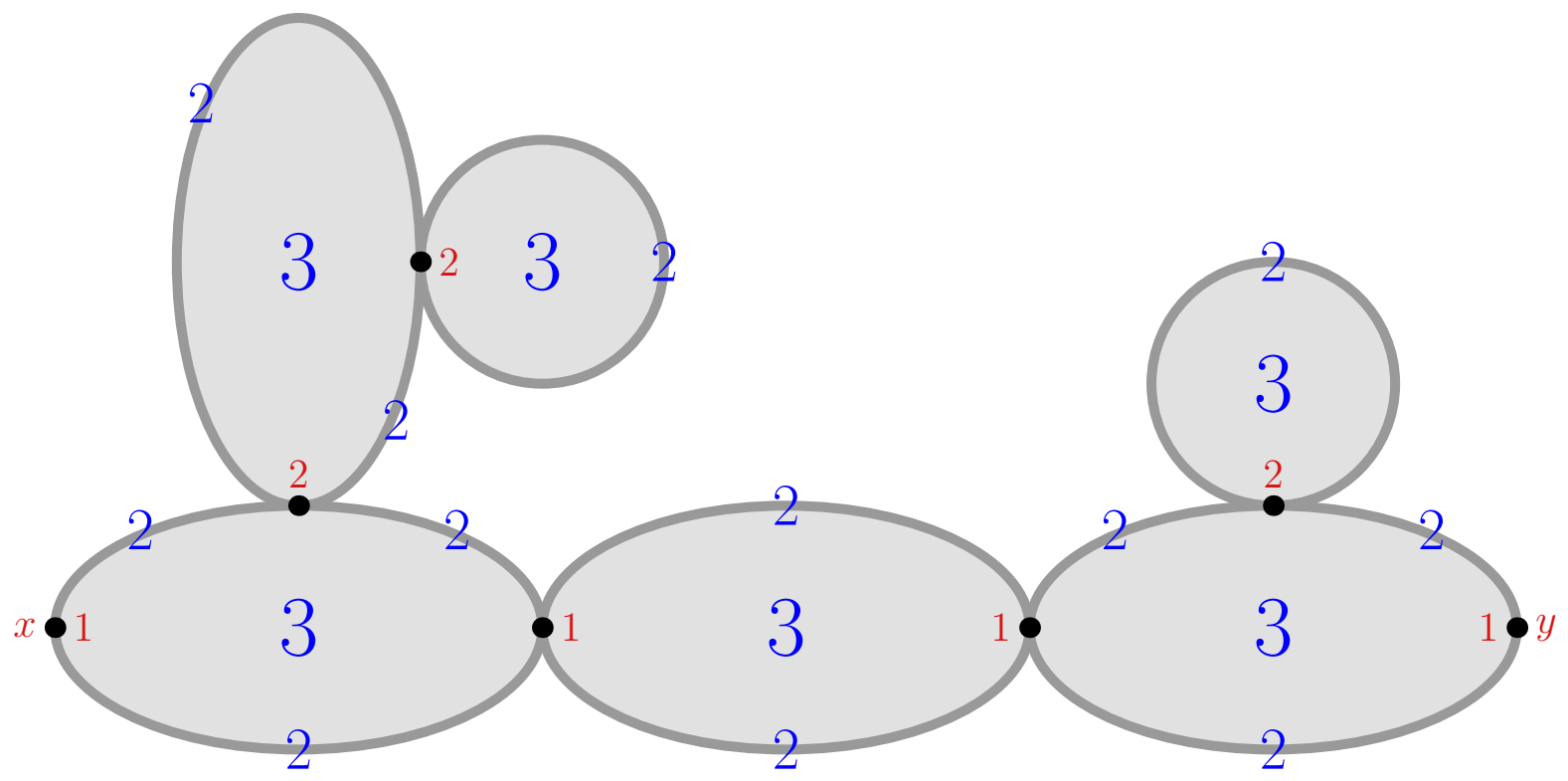

Figure 1: An illustration of the conditions in Lemma 2.2. The drawing represents a plane graph $G$, with each block of $G$ shown as an oval. Dots represent vertices $x$ and $y$, and cut vertices of $G$. Numbers give minimum list sizes: small red numbers for individual vertices shown as dots, larger blue numbers for classes of vertices.

Lemma 2.2. Let $G$ be a connected plane graph with distinguished vertices $x, y$-not necessarily distinct-lying on the outer face. Let each vertex $v$ of $G$ be assigned a list $L(v)$ of colors such that

$$
|L(v)| \geqslant \begin{cases}1, & \text { if } v \in S_{G}(x, y) \\ 2, & \text { if } v \text { is any other vertex on the outer face; } \\ 3, & \text { otherwise, i.e., if } v \text { is an interior vertex. }\end{cases}
$$

Then there exists a path coloring of $G$ in which each vertex receives a color from its list.

See Figure 1 for an illustration of the conditions on $G$ in Lemma 2.2.

Proof. We proceed by induction on the order of $G$. If $G$ has order 2 or less, then simply color each vertex with any color from its list. Now suppose that $G$ has order at least 3 . We may assume that each face of $G$ - except possibly the outer face - is a triangle (if not, then adding edges, coloring the resulting graph, and then removing the added edges will result in the required coloring of $G$ ).

Number the vertices on the outer face consecutively, going clockwise:

$$
x=z_{0}, z_{1}, \ldots, z_{k}=y, z_{k+1}, \ldots
$$

Note that if $v$ is a cut vertex of $G$, then $v$ appears in this list more than once.

Let $C_{x}$ be a color in $L(x)$. Consider the collection of all induced paths $P$ in $G$ such that $P$ begins at $x$ and ends in the set $\left\{z_{i}: 0 \leqslant i \leqslant k\right\}$ and every vertex of $P$ has $C_{x}$ in 
its list. This collection of paths is nonempty, since it contains the trivial path beginning and ending at $x=z_{0}$. Let $P_{0}$ be a path in this collection whose ending point has the greatest possible index $e \leqslant k$; so $z_{e}$ is the ending point of $P_{0}$.

See the upper graph in Figure 2 for an illustration.

Color each vertex of $P_{0}$ with color $C_{x}$. Create a new list assignment $L^{\prime}$ for vertices in $G-P_{0}$. If a vertex $v$ is adjacent in $G$ to a vertex of $P_{0}$, then let $L^{\prime}(v)=L(v)-\left\{C_{x}\right\}$; otherwise, let $L^{\prime}(v)=L(v)$.

We wish to apply the induction hypothesis to each component of $G-P_{0}$, using the $L^{\prime}$ lists. In each component we choose vertices $x^{\prime}$ and $y^{\prime}$ to take the role of $x$ and $y$, respectively, in the statement of the lemma; we then verify that the conditions of the lemma are met. The colors given by the lemma, along with the coloring of each vertex of $P_{0}$ with $C_{x}$, will give us the required path coloring of $G$ (since color $C_{x}$ has been removed from the list of each vertex of $G-P_{0}$ that is adjacent to a vertex of $P_{0}$ ).

Let $H$ be a component of $G-P_{0}$ with the plane embedding inherited from $G$. Let $i_{1}=\max \left\{i: z_{i} \in V(H)\right\}$, and let $x^{\prime}=z_{i_{1}}$. If $y \in V(H)$, then let $i_{2}=k$; otherwise, let $i_{2}=\min \left\{i: z_{i} \in V(H)\right\}$. Let $y^{\prime}=z_{i_{2}}$.

We make three observations concerning $x^{\prime}$ and $y^{\prime}$. First, if $y \in V(H)$, then $y^{\prime}=y$. Second, it is possible that $x^{\prime}=y^{\prime}$. Third, $x^{\prime}$ has a neighbor in $P_{0}$, as $z_{i_{1}+1}$ or $z_{0}$ if there is no $z_{i_{1}+1}$ in $G$-does not lie in $H$.

See the lower graph in Figure 2 for an illustration of $x^{\prime}$ and $y^{\prime}$ in components of $G-P_{0}$.

Graph $H$ is a connected plane graph, and vertices $x^{\prime}$ and $y^{\prime}$ lie on the outer face. In order to apply the induction hypothesis to $H$, it remains to show that the $L^{\prime}$ lists have the required sizes.

Let $v$ be a vertex of $H$ that does not lie on the outer face of $H$. Then $v$ does not lie on the outer face of $G$, so $|L(v)| \geqslant 3$. Vertex $v$ cannot be adjacent in $G$ to any vertex of $P_{0}$; thus $L^{\prime}(v)=L(v)$, and $\left|L^{\prime}(v)\right| \geqslant 3$.

Now let $w$ be a vertex of $H$ with $\left|L^{\prime}(w)\right| \leqslant 1$. We show that $\left|L^{\prime}(w)\right|=1$ and $w \in$ $S_{H}\left(x^{\prime}, y^{\prime}\right)$. This will suffice to verify that the $L^{\prime}$ lists have the required sizes, and will thus complete the proof of the lemma. We consider two cases. In Case I, $w \in S_{G}(x, y)$. In Case II, $w \notin S_{G}(x, y)$.

Case I. $w \in S_{G}(x, y)$.

Let $j=\min \left\{i: w=z_{i}\right\}$. We cannot have $j \leqslant e$, as then $w \in S_{G}\left(x, x_{e}\right)$, and $w$ would lie on $P_{0}$. The walk $x=z_{0}, z_{1}, \ldots, z_{k}=y$ in $G$ must hit every vertex in $S_{G}(x, y)$; so $j \leqslant k$.

Therefore, $e<j \leqslant k$. Suppose $\left|L^{\prime}(w)\right|=0$. Then $C_{x}$ was removed from $L(w)$ to obtain $L^{\prime}(w)$, so $w$ is adjacent to a vertex of $P_{0}$, and $C_{x} \in L(w)$. But this contradicts our choice of $P_{0}$ as the path whose ending point has the greatest possible index. By contradiction, $\left|L^{\prime}(w)\right|=1$.

Path $P_{0}$ must be contained in a single component of $G-w$ : the component containing $x$. Since $w \in S_{G}(x, y)$, vertex $y$ does not lie in this component. Therefore, there is a path in $G-P_{0}$ from $w$ to $y$, so $y \in H$. By our choice of $y^{\prime}$, we have $y^{\prime}=y$. Now consider a walk in $G$ beginning at $x$, following $P_{0}$ until reaching a neighbor of $x^{\prime}$, and then following a path in $H$ from $x^{\prime}$ to $y^{\prime}=y$. If $w \notin S_{H}\left(x^{\prime}, y^{\prime}\right)$, then we may choose this path to avoid $w$, 

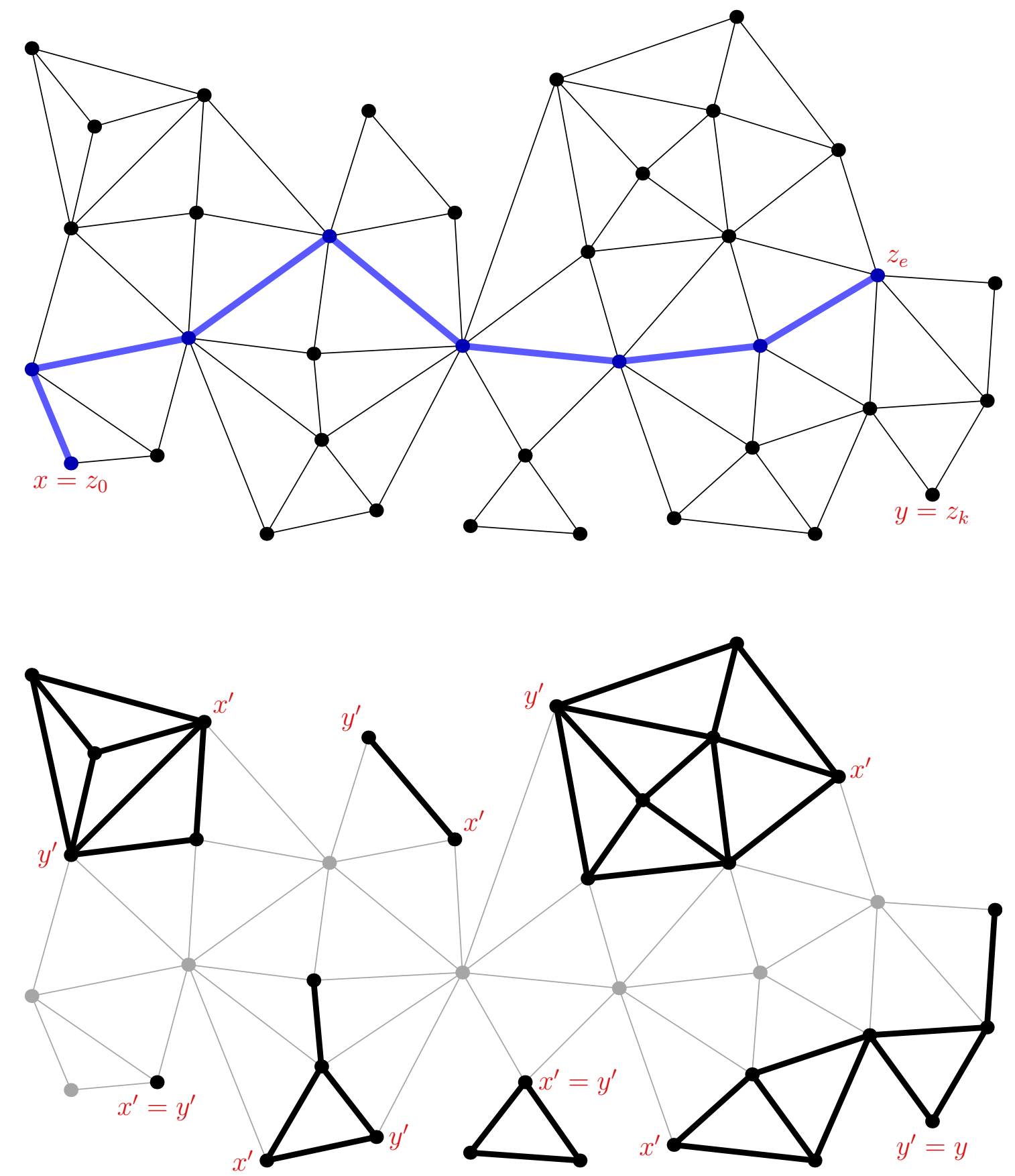

Figure 2: An illustration of part of the process involved in the proof of Lemma 2.2. Above is a plane graph $G$ with an induced path $P_{0}$ shown in blue. Vertices $x, y$, and $z_{e}$ are labeled. Below is the same graph $G$, with the components of $G-P_{0}$ highlighted. Vertices $x^{\prime}$ and $y^{\prime}$ are shown in each component. In the component containing $y$, we have $y^{\prime}=y$. 
and the walk in $G$ from $x$ to $y$ avoids $w$, contradicting the fact that $w \in S_{G}(x, y)$. Thus we must have $w \in S_{H}\left(x^{\prime}, y^{\prime}\right)$.

Case II. $w \notin S_{G}(x, y)$.

We have $|L(w)| \geqslant 2$, so $\left|L^{\prime}(w)\right| \geqslant 1$. We assumed that $\left|L^{\prime}(w)\right| \leqslant 1$, so $\left|L^{\prime}(w)\right|=1$.

We show that $w \in S_{H}\left(x^{\prime}, y^{\prime}\right)$. Suppose not. Then $w \neq x^{\prime}, w \neq y^{\prime}$, and there is a path in $H$ from $x^{\prime}$ to $y^{\prime}$ that avoids $w$. We will obtain a contradiction by exhibiting a $K_{5}$ minor in a planar graph $F$.

Let $F$ be a graph with vertex set $V\left(P_{0}\right) \cup V(H) \cup\left\{u_{1}, u_{2}\right\}$, where $u_{1}$ and $u_{2}$ are new vertices. Edges of $F$ will be all edges of $G$ with both endpoints in $V\left(P_{0}\right) \cup V(H)$, along with an edge from $u_{1}$ to $u_{2}$, an edge from $u_{1}$ to each of $x, y^{\prime}, w$, an edge from $u_{2}$ to each of $w, x^{\prime}, x$, and an edge from $w$ to $x^{\prime}$-unless such an edge exists in $H$.

We claim that $F$ is planar. Since $|L(w)| \geqslant 2$ and $\left|L^{\prime}(w)\right|=1$, color $C_{x}$ was removed from $L(w)$ to obtain $L^{\prime}(w)$; thus, $w$ is adjacent to a vertex of $P_{0}$, and $C_{x} \in L(w)$. Further, $|L(w)|=2$, so $w$ lies on the outer face of $G$. Let $i_{3}$ be the greatest integer such that $w=z_{i_{3}}$.

We show that $0<i_{2}<i_{3}<i_{1}$. None of $i_{1}, i_{2}, i_{3}$ can be zero, since $z_{0}=x$ lies on $P_{0}$, and so does not lie in $H$. Since $w \neq x^{\prime}, y^{\prime}$, we have $i_{3} \neq i_{1}, i_{2}$. By our choice of $x^{\prime}=z_{i_{1}}$, we have $i_{3}<i_{1}$. If $y \notin V(H)$, then, by our choice of $y^{\prime}$, we have $i_{2}<i_{3}$. Now suppose that $y \in V(H)$. Since $w$ is adjacent to a vertex of $P_{0}$ and $C_{x} \in L(w)$, we cannot have $e<i_{3}<k=i_{2}$, as this would contradict our choice of $P_{0}$ as the path whose ending point has the greatest possible index. We also cannot have $i_{3}<e$, as then $P_{0}$ would separate $w$ and $y$ in $G$, and these two vertices could not lie in the same component of $G-P_{0}$. Therefore, $i_{2}<i_{3}$. We conclude that, in all cases, $0<i_{2}<i_{3}<i_{1}$.

The subgraph of $G$ induced by $V\left(P_{0}\right) \cup V(H)$ is planar, since $G$ is planar. Since $x=z_{0}$, $y^{\prime}=z_{i_{2}}, w=z_{i_{3}}$, and $x^{\prime}=z_{i_{1}}$ lie on the outer face of $G$ in clockwise order, if we place vertices $u_{1}, u_{2}$ in the outer face of $G$, then we may add the edges involving $u_{1}$ and $u_{2}$, and the edge from $w$ to $x^{\prime}$, without crossing any existing edges, resulting in a plane embedding of $F$. Hence, the claim holds: $F$ is a planar graph.

To finish our proof, we obtain a contradiction by finding a $K_{5}$ minor in $F$. Start with graph $F$, and contract all edges of $P_{0}$. If $x^{\prime} \neq y^{\prime}$, then also contract all edges of any path in $H$ from $x^{\prime}$ to $y^{\prime}$ that avoids $w$. In the resulting graph, vertices $x, x^{\prime}$, and $w$ are all adjacent to each other, since $x^{\prime}$ and $w$ both have neighbors in $P_{0}$, and $x^{\prime}$ and $w$ are joined by an edge in $F$. In addition, vertices $u_{1}$ and $u_{2}$ are adjacent to each other and to all three of $x, x^{\prime}$, and $w$, since $x^{\prime}=y^{\prime}$ after contracting edges. We see that vertices $x, x^{\prime}, w$, $u_{1}$, and $u_{2}$ are distinct and all adjacent to each other; they thus induce a $K_{5}$.

Hence, we have a $K_{5}$ minor in $F$, a planar graph. By contradiction, $w \in S_{H}\left(x^{\prime}, y^{\prime}\right)$. This completes Case II and the proof of the lemma.

Our proof of Lemma 2.2 is constructive. An implementation of an efficient pathcoloring algorithm based on this proof is discussed in Bross, Chappell, \& Hartman [8].

Curiously, Theorem 2.1 has more or less been proven, while not being explicitly stated, in two other papers. As noted previously, Škrekovski [23, Thm. 2.2b] and Eaton \& Hull [12, Thm. 1] independently showed that every planar graph is 2-defective 3-choosable. Recall that, in the coloring associated with this property, each color class is a disjoint union of 
paths and cycles. In both cases, the proof is constructive, and the construction never actually produces cycles; it only produces paths - but this fact is not noted in either paper. The proof due to Škrekovski uses much the same technique as that in our proof above, while the Eaton-Hull proof is somewhat different.

Readers who have seen the proof by Škrekovski [23, pf. of Lemma 2.1] may object to our claim that that proof and our proof of Lemma 2.2 are much the same; the two proofs are written quite differently. However, the proofs involve a common underlying idea, which is as follows. Given distinguished vertices $x, y$, find a path that begins at $x$, ends on the outer face as far as possible toward $y$, and is colorable with a color available for $x$. Color this path with that color, remove that color from the lists of all neighbors of the path, remove the path from the graph, and invoke the induction hypothesis; and then everything else "just works."

The idea above is a simple one; the bulk of the effort in both proofs lies in showing that it "just works," and it is there that they differ. The proof of Škrekovski is written as a case analysis; some of the cases involve splitting the graph into blocks. Thus, when the path is removed, Škrekovski does not need to worry about cut vertices. But care must be taken to ensure that, when blocks are joined together, the coloring on the graph as a whole has the required properties. We, on the other hand, proceed in a single step. We avoid most of the cases, but we then need to spend time talking about cut vertices - our argument involving the $K_{5}$ minor.

We leave it to the reader to judge which form of the proof is preferable; we are not certain ourselves.

The following is an immediate corollary of Lemma 2.2 .

Theorem 2.3. If $G$ is an outerplanar graph, then $G$ is path 2-choosable.

Theorem 2.3 was proven by the second author in his Ph.D. thesis [17] (see the comments after the proof of Lemma 4.2 in that thesis). As with Theorem 2.1, Skrekovski [23, Thm. 2.2a] and Eaton \& Hull [12, Corollary 2] also proved a slightly weaker form of Theorem 2.3: that every outerplanar graph is 2-defective 2-choosable.

\section{Path List Multicoloring: Outerplanar Graphs}

Recall that a graph $G$ is $(q, r)$-choosable if, whenever each vertex of $G$ is assigned a list of $q$ colors, we may color each vertex with a set of $r$ colors from its list so that adjacent vertices are colored with disjoint sets.

The following question is due to Erdős, Rubin, \& Taylor [13, p. 155].

Question 3.1 (Erdős, Rubin, \& Taylor 1980). Let $q, r$, and $m$ be positive integers. If a graph $G$ is $(q, r)$-choosable, is $G$ necessarily $(m q, m r)$-choosable?

Little is known about the answer to Question 3.1 for graphs in general. Gutner [15, Thm. 1.16] proved that every $(2,1)$-choosable graph is $(4,2)$-choosable. Tuza \& Voigt [26, 
Thm. 1] showed that, if a graph is $(2,1)$-choosable, then it is $(2 m, m)$-choosable, for each positive integer $m$. See Gutner \& Tarsi [16] for a summary of these and related ideas.

It seems likely that Question 3.1 gets a "yes" answer, and that this idea generalizes to colorings of hypergraphs. Let $\mathcal{H}=(X, \mathcal{F})$ be a hypergraph with finite vertex set $X$ and hyperedge set $\mathcal{F}$. In a proper multicoloring of $\mathcal{H}$, each vertex of $\mathcal{H}$ is colored with a set of colors such that, for each hyperedge $e \in \mathcal{F}$, there is no color that is used on every vertex in $e$. Equivalently, for each color $C$, the set of vertices on which $C$ is used forms an independent set in $\mathcal{H}$, that is, a set $A$ of vertices of $\mathcal{H}$ such that $A$ contains no hyperedge of $\mathcal{H}$. We say $\mathcal{H}$ is $(q, r)$-choosable if, whenever each vertex of $\mathcal{H}$ is assigned a list of $q$ colors, there is a proper multicoloring of $\mathcal{H}$ in which each vertex receives a set of $r$ colors from its list.

Conjecture 3.2. Let $q, r$, and $m$ be positive integers. If a hypergraph $\mathcal{H}$ is $(q, r)$ choosable, then $\mathcal{H}$ is $(m q, m r)$-choosable.

Conjecture 3.2 is not really new, although we do not know that it has previously been published. Certainly, the conjecture is implicit in works like that of Mihók, Tuza, \& Voigt [20].

We mention hypergraph coloring because path coloring is a special case.

Observation 3.3. Let $G$ be a graph. Let $\mathcal{H}=(X, \mathcal{F})$ be the hypergraph with vertex set $X=V(G)$ whose hyperedges are those subsets of $V(G)$ that induce, in $G$, either a cycle or a claw $\left(K_{1,3}\right)$. Then the proper vertex colorings of $\mathcal{H}$ are precisely the path colorings of $G$, and the proper multicolorings of $\mathcal{H}$ are precisely the path multicolorings of $G$.

While we have not been able to prove Conjecture 3.2 in full generality, applying Conjecture 3.2 to the statement of Theorem 2.3 gives the following, which we can prove.

Theorem 3.4. If $G$ is an outerplanar graph, then $G$ is path $(2 r, r)$-choosable, for each positive integer $r$.

Theorem 3.4 follows from our next lemma. An outerplane graph is an outerplanar graph along with an embedding in the plane such that each vertex lies on the outer face.

Lemma 3.5. Let $G$ be an outerplane graph with distinguished vertices $x, y$, such that $x y$ is an edge lying on the outer face. Let $r$ be a positive integer. Let each vertex $v$ of $G$ be assigned a list $L(v)$ of colors such that

$$
|L(v)| \geqslant \begin{cases}r, & \text { if } v \in\{x, y\} \\ 2 r, & \text { if } v \text { is any other vertex. }\end{cases}
$$

Then there exists a path multicoloring of $G$ in which each vertex receives $r$ colors from its list, $x$ has no neighbor in any of its color classes, other than $y$, and $y$ has degree at most 1 in each of its color classes. 
Proof. We proceed by induction on the order of $G$. If $G$ has order 2 , then simply color each vertex with $r$ colors from its list. Now suppose that $G$ has order at least 3 .

We may assume that $G$ is maximal outerplanar; if not, add edges to $G$, maintaining an outerplane embedding and keeping edge $x y$ on the outer face. We may further assume that $|L(x)|=r$.

Vertices $x, y$ must have exactly one common neighbor; call it $z$. To see this, first observe that, if there are no common neighbors, then $x y$ is a cut edge, so $G$ is not maximal outerplanar. Second, if there is another common neighbor, say $z^{\prime}$, then, because edge $x y$ lies on the outer face, we can add a new vertex $z^{\prime \prime}$ adjacent to both $x$ and $y$, to obtain an outerplanar graph with a $K_{2,3}$ subgraph, which is impossible.

Create a new list assignment $L^{\prime}$. Let $L^{\prime}(z)$ be an $r$-element subset of $L(z)-L(x)$. For each vertex $v \in V(G)-\{z\}$, let $L^{\prime}(v)=L(v)$.

Let $G_{1}^{\prime}$ be the subgraph of $G$ induced by $x, z$, and each other vertex $v$ such that $\{x, z\}$ separates $v$ and $y$. Similarly, let $G_{2}^{\prime}$ be the subgraph of $G$ induced by $y, z$, and each other vertex $v$ such that $\{y, z\}$ separates $v$ and $x$. Note that $G_{1}^{\prime}$ and $G_{2}^{\prime}$ have exactly one vertex in common: $z$. We have $V\left(G_{1}^{\prime}\right) \cup V\left(G_{2}^{\prime}\right)=V(G)$; any vertex $v$ outside this union would be the endpoint of three paths reaching $x, y, z$, respectively, without meeting either of the other two vertices, resulting in a $K_{4}$ minor, which is impossible.

See Figure 3 for an illustration of $G_{1}^{\prime}$ and $G_{2}^{\prime}$.

Edge $x z$ lies on the outer face of $G_{1}^{\prime}$; apply the induction hypothesis to $G_{1}^{\prime}$, with distinguished vertices $x, z$ (playing the role of $x, y$, respectively) and list assignment $L^{\prime}$. Similarly, edge $y z$ lies on the outer face of $G_{2}^{\prime}$; apply the induction hypothesis to $G_{2}^{\prime}$, with distinguished vertices $y, z$ (playing the role of $x, y$, respectively) and list assignment $L^{\prime}$. These applications of the induction hypothesis will color all vertices, although vertex $z$, lying in both subgraphs, is colored twice. However, $\left|L^{\prime}(z)\right|=r$, so $z$ must receive the same $r$ colors in $G_{1}^{\prime}$ and in $G_{2}^{\prime}$. Thus, we have a vertex coloring of $G$.

It remains to show that this is the required path multicoloring.

Subgraphs $G_{1}^{\prime}$ and $G_{2}^{\prime}$ are both path multicolored, each vertex $v$ receives $r$ colors from $L^{\prime}(v)$, and $L^{\prime}(v) \subseteq L(v)$.

In $G_{1}^{\prime}, x$ has no neighbor in any of its color classes, other than $z$. However, $L^{\prime}(x) \cap$ $L^{\prime}(z)=\varnothing$. Thus, in $G, x$ has no neighbor in any of its color classes, other than $y$, as required.

In $G_{2}^{\prime}, y$ has no neighbor in any of its color classes, other than $z$. Vertex $y$ may also share a color with its neighbor $x$ in $G$. However, again, $L^{\prime}(x) \cap L^{\prime}(z)=\varnothing$, so no color used on $y$ can be used on both $x$ and $z$. Thus, in $G, y$ has degree at most 1 in each of its color classes, as required.

In each of $G_{1}^{\prime}$ and $G_{2}^{\prime}, z$ has degree at most 1 in each of its color classes. Thus, in $G$, $z$ has degree at most 2 in each of its color classes.

It remains to show that no color class containing a cycle is formed when $G_{1}^{\prime}$ and $G_{2}^{\prime}$ are glued together. The set $\{x, y, z\}$ separates all other vertices of $G_{1}^{\prime}$ from all other vertices of $G_{2}^{\prime}$, so any new cycle must contain at least 2 vertices of $\{x, y, z\}$. However, $x$ and $y$ each have degree at most 1 in each of their color classes, and so cannot lie in any cycle. 


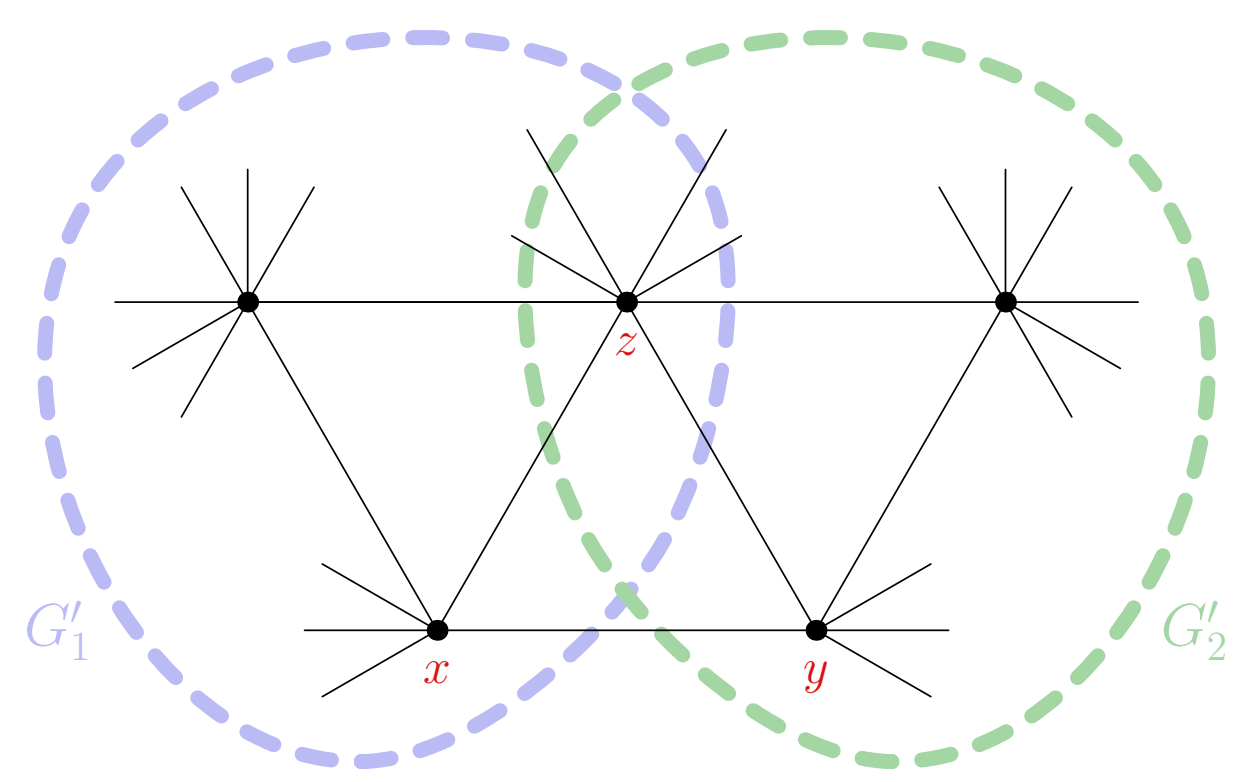

Figure 3: An illustration of part of the process involved in the proof of Lemma 3.5. Shown is a portion of a maximal outerplane graph $G$, with vertices $x, y$, and their unique common neighbor $z$ labeled, along with subgraphs $G_{1}^{\prime}$ and $G_{2}^{\prime}$.

We can show that Theorem 3.4 is best possible, in that the "2" in its statement cannot be replaced by any smaller real number.

Proposition 3.6. For each $\epsilon>0$, there exists an outerplanar graph $G$ such that, if $q$ and $r$ are positive integers with $q / r<2-\epsilon$, then $G$ is not path $(q, r)$-choosable.

Proof. Let $\epsilon>0$. Let $t \geqslant 2$ be an integer such that $2-\epsilon t \leqslant 0$. Let $G$ be the star $K_{1, t}$; note that $G$ is outerplanar. Suppose for a contradiction that $q$ and $r$ are positive integers with $q / r<2-\epsilon$, and $G$ is path $(q, r)$-choosable.

Assign lists of $q$ colors to the vertices of $G$ so that every vertex is assigned the same list. There exists a path multicoloring of $G$ in which each vertex is colored with $r$ colors from this list. Fix such a multicoloring.

Let $x$ be the center vertex of the star $G$, that is, the unique vertex that is not a leaf. We partition the colors into two kinds. First are those colors used on vertex $x$; there are $r$ of these. Second are those colors that are not used on vertex $x$; there are $q-r$ of these.

Star $G$ has $t$ leaves, colored with $r$ colors each, so the total number of colors used on the leaves, counting repetitions, is $r t$. Consider the contribution to this total from each of the two kinds of colors. Any color used on $x$ can be used on at most two leaves. So the contribution to the total from colors used on $x$ is at most $2 r$. There are $q-r$ colors that are not used on $x$; the contribution to the total from such colors is at most $t(q-r)$. Thus, we have the following.

$$
\begin{aligned}
t r & \leqslant 2 r+t(q-r) \\
& =2 r+t(q-2 r)+t r
\end{aligned}
$$


Since $q / r<2-\epsilon$ and $r>0$, we have $q<2 r-\epsilon r$, so $q-2 r<-\epsilon r$. Continuing:

$$
\begin{array}{ll}
<2 r-\epsilon r t+t r & \text { since } t>0 \\
=r(2-\epsilon t)+t r & \\
\leqslant r \cdot 0+t r & \text { by our choice of } t, \text { since } r>0 \\
=t r . &
\end{array}
$$

Thus, we have $t r<t r$. By contradiction, the desired result holds.

Another way to state Theorem 3.4 would be to say that if $q / r \geqslant 2$, then every outerplanar graph is path $(q, r)$-choosable. Proposition 3.6 shows that the converse also holds. Thus, we have proven the following.

Theorem 3.7. The following are equivalent, for positive integers $q, r$.

(i) Every outerplanar graph is path $(q, r)$-choosable.

(ii) $q / r \geqslant 2$.

\section{Path List Multicoloring: Planar Graphs}

We would like to prove results for planar graphs analogous to our list-multicoloring theorems for outerplanar graphs (Theorems 3.4 and 3.7). Every planar graph is path 3choosable (Theorem 2.1) and thus path $(3,1)$-choosable. Hence, Conjecture 3.2, if true, would imply that every planar graph is path $(3 r, r)$-choosable, for each positive integer $r$. However, we have been unable to prove this.

This is somewhat surprising. Tuza [25, Section 1.4] remarked that, "... almost all proofs showing that a certain graph is $(k, 1)$-choosable can be extended with little effort to verify $(k m, m)$-choosability." This observation generally extends to other kinds of graph coloring.

However, the technique used in our proof that planar graphs are path 3-choosable (Theorem 2.1) does not appear to work in the context of path multicolorings; nor does the technique used in the proof of Eaton \& Hull [12, pf. of Thm. 1] (see the comments after our proof of Lemma 2.2). We therefore leave generalized path choosability of planar graphs as an open problem.

Conjecture 4.1. If $G$ is a planar graph, then $G$ is path $(3 r, r)$-choosable, for each positive integer $r$.

We have not even been able to show that every planar graph is path $(6,2)$-choosable. However, we can show that Conjecture 4.1 is best possible, if it is true: the " 3 " in its statement cannot be replaced by any smaller real number. We use the following example, found in Cowen, Cowen, \& Woodall [11, pf. of Thm. 3] (and introduced there to prove something else). 


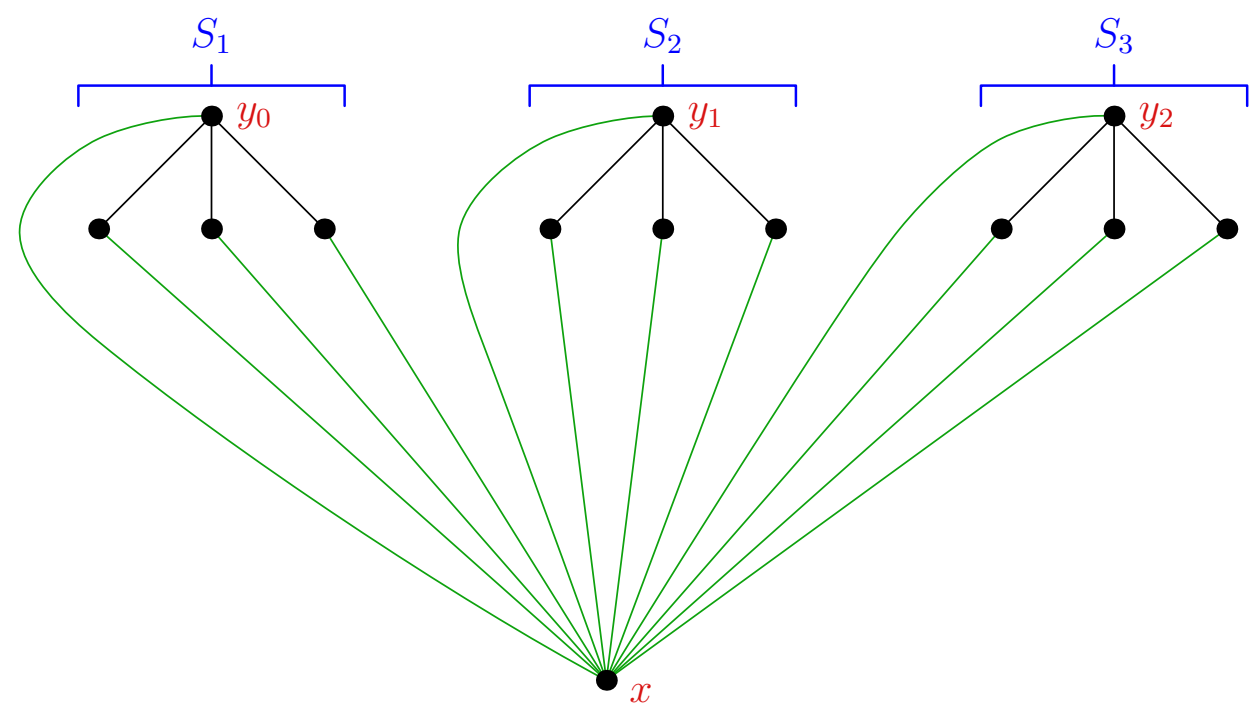

Figure 4: A plane embedding of graph $M_{3}$ from Example 4.2. Black edges are those contained in the stars $S_{1}, S_{2}$, and $S_{3}$. Green edges are those incident with vertex $x$.

Example 4.2. Given a positive integer $t$, define a planar graph $M_{t}$ as follows. Let $S_{1}, \ldots, S_{t}$ be $t$ disjoint copies of the star $K_{1, t}$. For each $i=1, \ldots, t$, let $y_{i}$ be the center vertex of star $S_{i}$, that is, the unique element of the partite set of cardinality 1 . Let $M_{t}$ be the join of a single new vertex $x$ with $S_{1} \cup \cdots \cup S_{t}$; that is, for each vertex $v \in S_{i}$ $(i=1, \ldots, t)$, there is an edge between $x$ and $v$. Since the disjoint union of stars is outerplanar, graph $M_{t}$ is a planar graph.

See Figure 4 for a drawing of graph $M_{3}$.

Proposition 4.3. For each $\epsilon>0$, there exists a planar graph $G$ such that, if $q$ and $r$ are positive integers with $q / r<3-\epsilon$, then $G$ is not path $(q, r)$-choosable.

Proof. Our proof is similar to that of Proposition 3.6.

Let $\epsilon>0$. Let $t \geqslant 3$ be an integer such that $-2+4 t-\epsilon t^{2} \leqslant 0$; such a $t$ must exist, since the leading coefficient of the left-hand side is negative. Let $G$ be the graph $M_{t}$ from Example 4.2; note that $G$ is planar. Suppose for a contradiction that $q$ and $r$ are positive integers with $q / r<3-\epsilon$, and $G$ is path $(q, r)$-choosable.

Assign lists of $q$ colors to the vertices of $G$ so that every vertex is assigned the same list. There exists a path multicoloring of $G$ in which each vertex is colored with $r$ colors from this list. Fix such a multicoloring.

Consider a star $S_{i}$ contained in $G$ (as defined in Example 4.2). Let $a_{i}$ be the number of colors used on vertex $x$ that are also used on vertex $y_{i}$, the center of star $S_{i}$. We partition the colors into three kinds. First are those colors used on vertex $x$; there are $r$ of these. Second are those colors used on vertex $y_{i}$ but not on $x$; there are $r-a_{i}$ of these. Third are those colors used on neither $x$ nor $y_{i}$; there are $q-r-\left(r-a_{i}\right)=q-2 r+a_{i}$ of these.

Star $S_{i}$ has $t$ leaves colored with $r$ colors each, so the total number of colors used on the leaves of $S_{i}$, counting repetitions, is $r t$. Consider the contribution to this total from 
each of the three kinds of colors. Let $b_{i}$ be the contribution to the total from colors used on vertex $x$. There are $r-a_{i}$ colors used on vertex $y_{i}$ but not $x$, and each of these can be used on at most 2 of the leaves of $S_{i}$, so the contribution to the total from such colors is at most $2\left(r-a_{i}\right)$. Lastly, there are $q-2 r+a_{i}$ colors used on neither $x$ nor $y_{i}$; the contribution to the total from such colors is at most $t\left(q-2 r+a_{i}\right)$. Thus, we have the following.

$$
t r \leqslant b_{i}+2\left(r-a_{i}\right)+t\left(q-2 r+a_{i}\right) .
$$

Now consider the total number of colors used on the leaves of all the $S_{i}$ stars, again counting repetitions. Summing the above, we obtain the following.

$$
\begin{aligned}
\sum_{i} t r & \leqslant \sum_{i}\left[b_{i}+2\left(r-a_{i}\right)+t\left(q-2 r+a_{i}\right)\right] \\
& =\sum_{i} b_{i}+2 t r+t^{2}(q-2 r)+(t-2) \sum_{i} a_{i}
\end{aligned}
$$

There are $r$ colors used on vertex $x$. Each of these may be used on at most 2 other vertices of $G$. Thus $\sum_{i} a_{i} \leqslant 2 r$, and similarly $\sum_{i} b_{i} \leqslant 2 r$. Continuing:

$$
\begin{array}{ll}
\leqslant 2 r+2 t r+t^{2}(q-2 r)+2 r(t-2) & \text { since } t-2>0 \\
=-2 r+4 t r+t^{2}(q-3 r)+t^{2} r &
\end{array}
$$

Since $q / r<3-\epsilon$ and $r>0$, we have $q<3 r-\epsilon r$, so $q-3 r<-\epsilon r$. Continuing again:

$$
\begin{aligned}
& <-2 r+4 t r-\epsilon r t^{2}+t^{2} r \quad \text { since } t^{2}>0 \\
& =r\left(-2+4 t-\epsilon t^{2}\right)+t^{2} r \\
& \leqslant r \cdot 0+t^{2} r \quad \text { by our choice of } t \text {, since } r>0 \\
& =\sum_{i} t r
\end{aligned}
$$

Thus, we have $\sum_{i} t r<\sum_{i} t r$. By contradiction, the desired result holds.

By Proposition 4.3, we see that Conjecture 4.1 is equivalent to the following.

Conjecture 4.4. The following are equivalent, for positive integers $q, r$.

(i) Every planar graph is path $(q, r)$-choosable.

(ii) $q / r \geqslant 3$.

That (i) implies (ii) in Conjecture 4.4 follows from Proposition 4.3. We have not been able to prove (ii) implies (i). However, our final result will be a slightly weaker form of this. Our proof will make use of results related to the Erdős-Rubin-Taylor question (Question 3.1).

A theorem of Alon [2, Thm. 6.1], which was based on work of Alon, Kleitman, Pomerance, Saks, \& Seymour [3], implies that, if a graph $G$ is $(q, r)$-choosable, then $G$ is 
$(m q, m r)$-choosable for infinitely many positive integers $m$. A slightly stronger result, due to Alon, Tuza, \& Voigt [4, Thm. 1.3], was generalized to other kinds of graph colorings by Mihók, Tuza, \& Voigt [20, Thm. 2]. See the surveys of Tuza [25, Section 3.4] and of Kratochvíl, Tuza, \& Voigt [19, Section 8.2] for summaries of these and related ideas.

Mihók, Tuza, \& Voigt [20, Thm. 4] further generalized these ideas to hypergraphs.

Recall that an independent set in a hypergraph $\mathcal{H}$ is a set $A$ of vertices of $\mathcal{H}$ such that $A$ contains no hyperedge of $\mathcal{H}$. A fractional coloring of $\mathcal{H}$ is a nonnegative real-valued function $\varphi$ on the set $\mathcal{A}$ of all independent sets of $\mathcal{H}$, such that, for each vertex $v$ of $\mathcal{H}$, we have

$$
\sum_{v \in A \in \mathcal{A}} \varphi(A) \geqslant 1
$$

The fractional chromatic number of $\mathcal{H}$, denoted by $\chi^{*}(\mathcal{H})$, is the infimum, over all fractional colorings $\varphi$ of $\mathcal{H}$, of

$$
\sum_{A \in \mathcal{A}} \varphi(A)
$$

Theorem 4.5 (Mihók, Tuza, \& Voigt 1999). For every positive integer $n$ there exists a number $f(n) \leqslant(n+1)^{2 n+2}$ such that the following holds. For every hypergraph $\mathcal{H}$ with $n$ vertices and fractional chromatic number $\chi^{*}(\mathcal{H})$, and for every positive integer $q$ that is divisible by all positive integers up to $f(n), \mathcal{H}$ is $(q, r)$-choosable where $r=q / \chi^{*}(\mathcal{H})$.

Theorem 4.5 has the following corollary, which almost, but not quite, verifies Conjecture 3.2. (As with Conjecture 3.2, this corollary is not really new, although we do not know that it has previously been published.)

Corollary 4.6. Let $q$ and $r$ be positive integers. If a hypergraph $\mathcal{H}$ is $(q, r)$-choosable, then there exist an infinite number of positive integers $m$ such that $\mathcal{H}$ is $(m q, m r)$-choosable.

Proof. Let $q$ and $r$ be positive integers, and let $\mathcal{H}$ be a $(q, r)$-choosable hypergraph. If we assign all vertices of $\mathcal{H}$ the same list of $q$ colors, and color from these lists, then we obtain a proper multicoloring of $\mathcal{H}$ in which each vertex is colored with $r$ colors, and a total of at most $q$ colors are used.

Define a fractional coloring $\varphi$ on $\mathcal{H}$. For each color $C$, the set of vertices on which $C$ is used in the above multicoloring forms an independent set in $\mathcal{H}$. Let $\varphi(A)=1 / r$ if $A$ is an independent set of this form, and $\varphi(A)=0$ otherwise.

For each vertex $v$ of $\mathcal{H}$, there are $r$ colors used on $v$, so we have

$$
\sum_{v \in A \in \mathcal{A}} \varphi(A)=r \cdot \frac{1}{r}=1 .
$$

Thus $\varphi$ is a fractional coloring of $\mathcal{H}$. There are exactly $q$ colors available to be used on vertices of $\mathcal{H}$, so

$$
\chi^{*}(\mathcal{H}) \leqslant \sum_{A \in \mathcal{A}} \varphi(A)=q \cdot \frac{1}{r} .
$$


Let $f(n)$ be as in the statement of Theorem 4.5, where $n$ is the order of $\mathcal{H}$. Let $s$ be a positive integer that is divisible by all positive integers up to $f(n)$. Let $t$ be a positive integer such that $t \cdot \chi^{*}(\mathcal{H})$ is an integer. Given a positive integer $k$, let $m=k s t$. Since there are an infinite number of such integers $m$ - one for each positive integer $k$-it suffices to show that $\mathcal{H}$ is $(m q, m r)$-choosable.

The value $m r \cdot \chi^{*}(\mathcal{H})$ is an integer, since $t \cdot \chi^{*}(\mathcal{H})$ is an integer. Further, $m r \cdot \chi^{*}(\mathcal{H})$ is divisible by all positive integers up to $f(n)$. Thus, by Theorem 4.5 , hypergraph $\mathcal{H}$ is $\left(m r \cdot \chi^{*}(\mathcal{H}), m r\right)$-choosable.

Since $\chi^{*}(\mathcal{H}) \leqslant q / r$, we have $m q \geqslant m r \cdot \chi^{*}(\mathcal{H})$. We conclude that $\mathcal{H}$ is $(m q, m r)$ choosable.

Applying Corollary 4.6 to the hypergraph of Observation 3.3, we obtain our final result.

Theorem 4.7. If $G$ is a planar graph, then there exist an infinite number of positive integers $m$ such that $G$ is path $(3 m, m)$-choosable.

Proof. Let $G$ be a planar graph. Let $\mathcal{H}$ be the hypergraph of Observation 3.3, whose vertices are the vertices of $G$, and whose hyperedges are the subsets of $V(G)$ inducing, in $G$, either a cycle or a claw $\left(K_{1,3}\right)$. As noted in Observation 3.3, the proper multicolorings of $\mathcal{H}$ are precisely the path multicolorings of $G$.

By Theorem 2.1, $G$ is path 3 -choosable, so $\mathcal{H}$ is $(3,1)$-choosable. By Corollary 4.6, there exist an infinite number of positive integers $m$ such that $\mathcal{H}$ is $(3 m, m)$-choosable. For each such $m, G$ is path $(3 m, m)$-choosable.

\section{References}

[1] J. Akiyama, H. Era, S. V. Gervacio, and M. Watanabe, Path chromatic numbers of graphs, J. Graph Theory 13 (1989), no. 5, 569-575. doi:10.1002/jgt.3190130506

[2] N. Alon, Restricted colorings of graphs, in Surveys in Combinatorics, 1993, London Math. Soc. Lecture Note Ser. 187, pp. 1-33, Cambridge Univ. Press., Cambridge, 1993.

[3] N. Alon, D. J. Kleitman, C. Pomerance, M. Saks, and P. Seymour, The smallest $n$ uniform hypergraph with positive discrepancy, Combinatorica 7 (1987), no. 2, 151160. doi:10.1007/BF02579446

[4] N. Alon, Zs. Tuza, and M. Voigt, Choosability and fractional chromatic numbers, Discrete Math. 165/166 (1997), 31-38. doi:10.1016/S0012-365X(96)00159-8

[5] M. Axenovich, T. Ueckerdt, and P. Weiner, Splitting planar graphs of girth 6 into two linear forests with short paths, J. Graph Theory 85 (2017), no. 3, 601-618. doi:10.1002/jgt. 22093

[6] O. V. Borodin and A. O. Ivanova, List strong linear 2-arboricity of sparse graphs, J. Graph Theory 67 (2011), no. 2, 83-90. doi:10.1002/jgt.20516 
[7] I. Broere and C. M. Mynhardt, Generalized colorings of outerplanar and planar graphs, in Graph theory with applications to algorithms and computer science (Kalamazoo, Mich., 1984), pp. 151-161, Wiley-Intersci. Publ., Wiley, New York, 1985.

[8] A. Bross, G. G. Chappell, and C. Hartman, Path-coloring algorithms for plane graphs, manuscript, 2018.

[9] G. G. Chappell, J. Gimbel, and C. Hartman, Thresholds for path coloring of planar graphs, in Topics in Discrete Mathematics, Algorithms Combin. 26, pp. 435-454, Springer, Berlin, 2006. doi:10.1007/3-540-33700-8_21

[10] G. Chartrand and H. V. Kronk, The point-arboricity of planar graphs, J. London. Math. Soc. 44 (1969), no. 1, 612-616. doi:10.1112/jlms/s1-44.1.612

[11] L. J. Cowen, R. H. Cowen, and D. R. Woodall, Defective colorings of graphs in surfaces: partitions into subgraphs of bounded valency, J. Graph Theory 10 (1986), no. 2, 187-195. doi:10.1002/jgt.3190100207

[12] N. Eaton and T. Hull, Defective list colorings of planar graphs, Bull. Inst. Combin. Appl. 25 (1999), 79-87.

[13] P. Erdős, A. L. Rubin, and H. Taylor, Choosability in graphs, in Proceedings of the West Coast Conference on Combinatorics, Graph Theory and Computing (Humboldt State Univ., Arcata, Calif., 1979), pp. 125-157, Congress. Numer. XXVI, Utilitas Math., Winnipeg, Man., 1980.

[14] W. Goddard, Acyclic colorings of planar graphs, Discrete Math. 91 (1991), no. 1, 91-94. doi : 10 . 1016/0012-365X (91)90166-Y

[15] S. Gutner, Choice Numbers of Graphs, M.Sc. thesis, Tel Aviv University, 1992.

[16] S. Gutner and M. Tarsi, Some results on ( $a: b)$-choosability, Discrete Math. 309 (2009), no. 8, 2260-2270. doi:10.1016/j.disc.2008.04.061

[17] C. M. Hartman, Extremal Problems in Graph Theory, Ph.D. thesis, University of Illinois, 1997.

[18] F. Havet and J.-S. Sereni, Improper choosability of graphs and maximum average degree, J. Graph Theory 52 (2006), no. 3, 181-199. doi:10.1002/jgt.20155

[19] J. Kratochvíl, Zs. Tuza, and M. Voigt, New trends in the theory of graph colorings: Choosability and list coloring, in Contemporary Trends in Discrete Mathematics: from DIMACS and DIMATIA to the future (R. L. Graham, J. Kratochvíl, J. Nešetřil, F. S. Roberts eds.), DIMACS Series in Discrete Mathematics and Theoretical Computer Science 49, pp. 183-197, American Mathematical Society, Providence, RI, 1999.

[20] P. Mihók, Zs. Tuza, and M. Voigt, Fractional $\mathcal{P}$-colourings and $\mathcal{P}$-choice-ratio, Tatra Mt. Math. Publ. 18 (1999), 69-77.

[21] P. Ossona de Mendez, S.-I. Oum, and D. R. Wood, Defective coloring of graphs excluding a subgraph or minor, Combinatorica (2018), advance online publication, 34 pp. doi:10.1007/s00493-018-3733-1 
[22] K. S. Poh, On the linear vertex-arboricity of a planar graph, J. Graph Theory 14 (1990), no. 1, 73-75. doi:10.1002/jgt.3190140108

[23] R. Škrekovski, List improper colourings of planar graphs, Combin. Probab. Comput. 8 (1999), no. 3, 293-299. doi:10.1017/S0963548399003752

[24] R. Škrekovski, List improper colourings of planar graphs with prescribed girth, Discrete Math. 214 (2000), no. 1-3, 221-233. doi:10.1016/S0012-365X(99)00145-4

[25] Zs. Tuza, Graph coloring with local constraints - a survey, Discuss. Math. Graph Theory 17 (1997), no. 2, 161-228.

[26] Zs. Tuza and M. Voigt, Every 2-choosable graph is $(2 m, m)$-choosable, J. Graph Theory 22 (1996), no. 3, 245-252.

[27] D. B. West, Introduction to Graph Theory, 2nd ed., Prentice Hall, Upper Saddle River, NJ, 2000.

[28] D. R. Wood, Defective and clustered graph colouring, Electron. J. Combin., dynamic survey \#DS23, April 13, 2018, 71 pp.

[29] D. R. Woodall, Defective choosability of graphs in surfaces, Discuss. Math. Graph Theory 31 (2011), no. 3, 441-459. doi:10.7151/dmgt. 1557 